TRANSACTIONS OF THE

AMERICAN MATHEMATICAL SOCIETY

Volume 358, Number 9, September 2006, Pages 4057-4070

S $0002-9947(05) 03804-3$

Article electronically published on September 22, 2005

\title{
SCOTT'S RIGIDITY THEOREM FOR SEIFERT FIBERED SPACES; REVISITED
}

\author{
TERUHIKO SOMA
}

\begin{abstract}
We will present a new proof of the rigidity theorem for Seifert fibered spaces of infinite $\pi_{1}$ by Scott (1983) in the case when the base of the fibration is a hyperbolic triangle 2-orbifold. Our proof is based on arguments in the rigidity theorem for hyperbolic 3-manifolds by Gabai (1997).
\end{abstract}

It has been twenty years since Scott [11] proved the rigidity theorem for Seifert fibered spaces $M$ with infinite fundamental groups. In fact, he proved the theorem in the case when the base of $M$ is a triangle 2-orbifold $O(p, q, r)$ with $1 / p+1 / q+$ $1 / r \leq 1$. In all other cases, $M$ is a Haken manifold and the proof of the theorem reduces to Waldhausen's rigidity theorem [14. Scott's result was the first major step in the proof of the Seifert Fibered Space Theorem which was completed by Gabai [5] and Casson-Jungreis [3] independently. The theorem says that if the fundamental group $\pi_{1}(M)$ of a closed irreducible 3-manifold $M$ contains a normal subgroup isomorphic to $\mathbf{Z}$, then $M$ is a Seifert fibered space. On the other hand, the rigidity theorem for hyperbolic 3-manifolds was proved by Gabai [6] in the case when manifolds satisfy the insulator condition. After that, Gabai, Meyerhoff and N. Thurston [7] completed the hyperbolic rigidity theorem by showing that any closed hyperbolic 3-manifold satisfies this condition.

In this paper, we will present a new proof of Scott's Rigidity Theorem in the case when the base $O$ of a Seifert fibered space $M$ is a hyperbolic triangle 2-orbifold. Roughly speaking, our proof is the 2-dimensional (and hence a simpler) version of Gabai's argument in [6]. Such an approach is very natural and compatible with the widely recognized idea in three-manifold topology that any Seifert fibered structure with infinite $\pi_{1}$ is a degenerating limit of 3-dimensional hyperbolic structures. In fact, the covering $\hat{X}$ of $M$ associated to an infinite cyclic normal subgroup of $\pi_{1}(M)$ is quasi-isometric to the hyperbolic plane $\mathbf{H}^{2}$. Our proof is completed by showing that the $\pi_{1}^{\text {orb }}(O)$-orbit of some point $x \in \mathbf{H}^{2}$ satisfies the 2-dimensional insulator condition.

Theorem 0.1. Let $f: M \longrightarrow N$ be a homotopy equivalence between closed orientable and irreducible 3-manifolds. If $M$ admits a Seifert-fibration whose base is a hyperbolic triangle 2-orbifold, then $f$ is homotopic to a homeomorphism.

As a benefit of our proof of Theorem 0.1, we have a considerably shorter proof of the following, which is also the hyperbolic base case of the theorem given by Scott 12] (see also Boileau-Otal [2]).

Received by the editors February 14, 2003 and, in revised form, June 28, 2004. 2000 Mathematics Subject Classification. Primary 57M99; Secondary 57M50.

Key words and phrases. Seifert fibered spaces, Scott's rigidity theorem, insulator condition.

(C)2005 American Mathematical Society Reverts to public domain 28 years from publication 4057 
Theorem 0.2. Let $f: M \longrightarrow M$ be a self-homeomorphism of a closed orientable Seifert fibered space with a hyperbolic base 2-orbifold. If $f$ is homotopic to the identity $\operatorname{Id}_{M}$ of $M$, then $f$ is isotopic to $\operatorname{Id}_{M}$.

\section{2- AND 3-DIMENSIONAL INSULATOR THEORIES; COMPARISON}

First of all, we will briefly review Gabai's insulator theory for hyperbolic 3manifolds, which will be helpful to grasp our analogous approach for Seifert fibered spaces.

Hyperbolic case. Suppose that $f: M \longrightarrow N$ is a homotopy equivalence from a closed hyperbolic 3-manifold $M$ to a closed irreducible 3-manifold $N$. Let $\rho$ be the hyperbolic metric on $M$, and let $\nu$ be a fixed Riemannian metric on $N$. It is well known that there exist finite coverings $p_{1}: X \longrightarrow M, q_{1}^{\prime}: X^{\prime} \longrightarrow N$ such that $f$ is lifted to a map $f_{1}^{\prime}: X \longrightarrow X^{\prime}$ which is homotopic to a homeomorphism $f_{1}$. By identifying $X$ with $X^{\prime}$ via $f_{1}$, we have the following homotopically commutative diagram, where $q_{1}=q_{1}^{\prime} \circ f_{1}$ and $X_{\rho}$ (resp. $X_{\nu}$ ) is supposed to have the Riemannian metric, still denoted by $\rho$ (resp. $\nu$ ), induced via $p_{1}$ (resp. $q_{1}$ ):

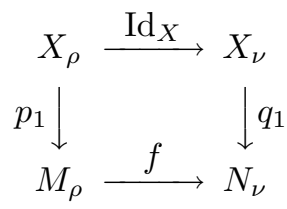

Let $p: \mathbf{H}_{\rho}^{3} \longrightarrow M_{\rho}, q: \mathbf{H}_{\sigma}^{3} \longrightarrow N_{\nu}$ be the universal coverings. Unfortunately, the definition of the insulator condition for hyperbolic 3-manifolds in [6] is not so simple, and it might be hard for us to take the notion at once. However, a striking theorem in [7] guarantees that any closed hyperbolic 3-manifold satisfies the condition. Roughly speaking, the insulator condition is used to designate an ambient isotopy class of $p_{1}^{-1}(c)$ in $X$, where $c$ is a core of some open solid torus $V$ in $M$. In fact, this condition gives a certain family $\left\{\lambda_{i: j}\right\}$ of simple loops in $\partial \mathbf{H}^{3}$ such that each component $V_{i}$ of $p^{-1}(V)$ is the 'central' component of $\mathbf{H}^{3}-\bigcup_{j \neq i} D_{i: j[\rho]}$, where $D_{i: j[\rho]}$ is a $\rho$-least area plane properly embedded in $\mathbf{H}_{\rho}^{3}$ with $\partial D_{i: j[\rho]}=\lambda_{i: j}$. In the original paper [6], $D_{i: j[\rho]}$ was a $D^{2}$-limit lamination in $\mathbf{H}_{\rho}^{3}$ spanned by $\lambda_{i: j}$. It was later shown that $D_{i: j[\rho]}$ can be supposed to be a properly embedded plane; see 13. We note that intersections of properly embedded planes can be treated fairly easily compared with those of $D^{2}$-limit laminations. By studying the situation of $D_{i: j\left[\nu_{t}\right]}$ under a continuous deformation of Riemannian metrics $\nu_{t}(0 \leq t \leq 1)$ on $X$ with $\nu_{0}=\rho, \nu_{1}=\nu$, one can see that $p_{1}^{-1}(c)$ is ambient isotopic in $X$ to $q_{1}^{-1}\left(c^{\prime}\right)$ for a core $c^{\prime}$ of some open solid torus $V^{\prime}$ in $N$. By using this fact, it is shown that $f$ is homotopic to a map $g: M \longrightarrow N$ such that $g^{-1}\left(V^{\prime}\right)=V$ and $\psi=g \mid(M-V): M-V \longrightarrow N-V^{\prime}$ is a homotopy equivalence; see [6] for details. By Waldhausen's Rigidity Theorem [14, $\psi$ is properly homotopic to a homeomorphism, which can be extended to a homeomorphism from $M$ to $N$ homotopic to $f$.

Next, suppose that $f: M \longrightarrow M$ is a self-homeomorphism of a closed hyperbolic 3 -manifold homotopic to the identify $\operatorname{Id}_{M}$. Let $V$ be an open solid torus designated by the insulator condition for $M$ with respect to the hyperbolic metric $\rho$ on $M$. Then, $f(V)$ is a designated open solid torus with respect to the push-forward metric $f_{*}(\rho)$ on $M$. It follows that $V$ is ambient isotopic to $f(V)$, and hence $f$ is isotopic 
to a homeomorphism $f^{\prime}: M \longrightarrow M$ with $f^{\prime} \mid V=\mathrm{Id}_{V}$. By using this fact, one can show that $f^{\prime}$ and hence $f$ are isotopic to $\operatorname{Id}_{M}$.

Seifert fibered case. Let $M$ be a closed Seifert fibered space with a hyperbolic base 2-orbifold $O$. For the proofs of Theorems 0.1 and 0.2 , it suffices to consider the case when $O$ is a triangle 2-orbifold.

Let $f: M \longrightarrow N$ be a homotopy equivalence from the manifold $M$ to a closed irreducible 3-manifold $N$. There exist finite coverings $p_{1}: X \longrightarrow M, q_{1}^{\prime}: X^{\prime} \longrightarrow N$ such that the induced fibration on $X$ is an $S^{1}$-bundle over a closed hyperbolic surface, and $f$ is lifted to a map $f_{1}^{\prime}$ homotopic to a homeomorphism $f_{1}: X \longrightarrow X^{\prime}$. Then, $q_{1}=q_{1}^{\prime} \circ f_{1}: X \longrightarrow N$ is also a finite covering. Let $p: \hat{X} \longrightarrow M$, $q: \hat{X} \longrightarrow N$ be the coverings associated to $\langle\gamma\rangle \subset \pi_{1}(M), f_{*}(\langle\gamma\rangle) \subset \pi_{1}(N)$, respectively. Both $G=\pi_{1}(M) /\langle\gamma\rangle$ and $G^{\prime}=\pi_{1}(N) / f_{*}(\langle\gamma\rangle)$ can be regarded as covering transformation groups on $\hat{X}$. Let $\hat{\sigma}: \hat{X} \longrightarrow \mathbf{H}^{2}$ be the projection of the induced $S^{1}$-bundle structure. Note that the isometric action of $G=\pi_{1}^{\text {orb }}(O)$ on $\mathbf{H}^{2}$ commutes the action of $G$ on $\hat{X}$ via $\hat{\sigma}$. Suppose that $\hat{X}$ has the Riemannian metric $\nu$ induced from a fixed Riemannian metric on $N$, which is also denoted by $\nu$. Then, $G^{\prime}$ acts on $\hat{X}$ isometrically. The definition of the 2 -dimensional insulator condition is fairly simple compared with the 3 -dimensional case, and it gives a certain family $\left\{\alpha_{i: j}\right\}$ of geodesic lines in $\mathbf{H}^{2}$ with $G\left(\bigcup_{i, j} \alpha_{i: j}\right)=\bigcup_{i, j} \alpha_{i: j}$. The proof of Lemma 2.3 which shows that any hyperbolic triangle group satisfies the insulator condition is short and elementary. By using the condition, we will prove that there exist $G^{\prime}$-equivariant, mutually disjoint open solid tori $\hat{V}_{i}$ in $\hat{X}$. Here, each $\hat{V}_{i}$ is the 'central' component of $\hat{X}-\bigcup_{j \neq i} A_{i: j}$, where $A_{i: j}$ is a $\nu$-least area open annulus properly homotopic in $\hat{X}$ to the open annulus $\hat{\sigma}^{-1}\left(\alpha_{i: j}\right)$. Theorem 0.1 asserts that the map $f$ is homotopic to a homeomorphism. In the proof, we do not need any deformation of Riemannian metrics on $X$. We just need the fact that $\hat{X}-\bigcup_{i} \operatorname{Int} \hat{W}_{i}$ is homeomorphic to $R \times S^{1}$ for some planar surface $R$, where $\hat{W}_{i}$ is a $G^{\prime}$-equivariant, solid torus core of $\hat{V}_{i}$. This implies that $N$ admits a Seifert fibration, and hence $f$ is homotopic to a homeomorphism.

Theorem 0.2 asserts that a self-homeomorphism $f: M \longrightarrow M$ is isotopic to $\operatorname{Id}_{M}$ if $f$ is homotopic to $\operatorname{Id}_{M}$. It will be proved by arguments similar to those in the hyperbolic case. In particular, we need to study the situation of designated solid tori $\hat{V}_{i}$ in $\hat{X}$ under a continuous deformation of Riemannian metrics on $M$ from $\nu$ to $f_{*}(\nu)$.

\section{INSULATOR CONDITION FOR FUCHSIAN ORBITS}

We refer to Hempel 8 and Jaco 9 ] for the fundamental notation and definitions of 3-manifold topology. All 3-manifolds in the paper are assumed to be orientable.

Let $M$ be a closed Seifert fibered space with the Seifert fibration $\sigma: M \longrightarrow O$, and let $f: M \longrightarrow N$ be a homotopy equivalence to a closed irreducible 3-manifold $N$. Suppose that the base $O$ is the hyperbolic triangle orbifold $O(p, q, r)$, where $p, q, r$ are integers with $2 \leq p \leq q \leq r$ and $1 / p+1 / q+1 / r<1$. Let $\langle\gamma\rangle$ be the cyclic subgroup of $\pi_{1}(M)$ such that the generator $\gamma$ is represented by a regular fiber of $M$. Since $O$ is orientable, $\langle\gamma\rangle$ is contained in the center $Z\left(\pi_{1}(M)\right)$ of $\pi_{1}(M)$. We set $G=\pi_{1}(M) /\langle\gamma\rangle$ and $G^{\prime}=\pi_{1}(N) /\left\langle f_{*}(\gamma)\right\rangle$, and let $\bar{f}_{*}: G \longrightarrow G^{\prime}$ be the isomorphism induced from $f_{*}: \pi_{1}(M) \longrightarrow \pi_{1}(N)$. The group $G$ is naturally identified with the orbifold fundamental group $\pi_{1}^{\text {orb }}(O)$. For any $g \in G$, the element 
$\bar{f}_{*}(g) \in G^{\prime}$ will be denoted by $g^{\prime}$. Let $a: F \longrightarrow O$ be an orbifold covering such that $F$ is a closed hyperbolic surface, and let $\hat{a}: \mathbf{H}^{2} \longrightarrow F$ be the universal covering. Then, $\pi_{1}(F)=\pi_{1}^{\text {orb }}(F)$ is regarded as a subgroup of $G=\pi_{1}^{\text {orb }}(O)$. Consider the finite covering $p_{1}: X \longrightarrow M$ associated to $\varphi^{-1}\left(\pi_{1}(F)\right) \subset \pi_{1}(M)$, where $\varphi: \pi_{1}(M) \longrightarrow G$ is the natural quotient epimorphism. If $q_{1}^{\prime}: X^{\prime} \longrightarrow N$ is the finite covering corresponding to $p_{1}$, then $f: M \longrightarrow N$ can be lifted to a homotopy equivalence $f_{1}^{\prime}: X \longrightarrow X^{\prime}$. Note that $X$ has the $S^{1}$-bundle structure $\sigma_{X}: X \longrightarrow F$ induced from $\sigma: M \longrightarrow O$. Since $X$ is a Haken manifold and $X^{\prime}$ is irreducible (see Meeks-Simon-Yau [10]), $f_{1}^{\prime}$ is homotopic to a homeomorphism $f_{1}$. Thus, we have the following commutative diagram:

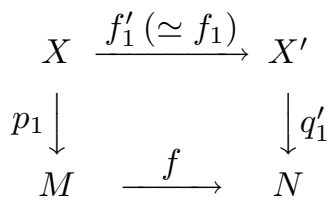

We will identify $X$ with $X^{\prime}$ via the homeomorphism $f_{1}$. If $\hat{p}: \hat{X} \longrightarrow X$ is the covering associated to $\langle\gamma\rangle \subset \pi_{1}(X)$, then $p_{1} \circ \hat{p}: \hat{X} \longrightarrow M, q_{1} \circ \hat{p}: \hat{X} \longrightarrow N$ are also coverings, where $q_{1}=q_{1}^{\prime} \circ f_{1}$.

Fix a Riemannian metric $\nu$ on $N$, and also denote the induced metrics on $X$ and $\hat{X}$ by $\nu$. The manifold $\hat{X}$ has the $S^{1}$-bundle structure $\hat{\sigma}: \hat{X} \longrightarrow \mathbf{H}^{2}$ induced from $\sigma_{X}$. Then, we have the following diagram consisting of four blocks. The right lower block is homotopically commutative, and the other three are commutative in the usual sense.

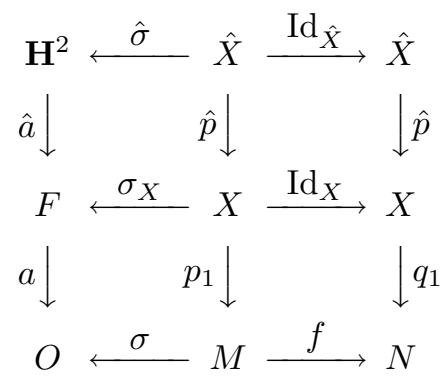

The group $G$ is regarded as an isometric and properly discontinuous transformation group on $\mathbf{H}^{2}$, i.e. $G$ is a Fuchsian group, and also the covering tranformation group on $\hat{X}$ with respect to $p_{1} \circ \hat{p}$. The group $G^{\prime}$ is regarded as the $\nu$-isometric tranformation group on $\hat{X}$ with respect to $q_{1} \circ \hat{p}$. Since the $\nu$-lengths of $S^{1}$-fibers $\hat{\sigma}^{-1}(x)\left(x \in \mathbf{H}^{2}\right)$ are bounded, $\hat{\sigma}: \hat{X} \longrightarrow \mathbf{H}^{2}$ is a quasi-isometry. In particular, one can suppose that the compactification of $\hat{X}$ as a Gromov hyperbolic space has the boundary $S_{\infty}^{1}$. The covering transformations of $\hat{X}$ corresponding to $g \in G$ and $g^{\prime} \in G^{\prime}$ are properly homotopic to each other such that the length of each trace of the homotopy is uniformly bounded. This means that the action of $G$ on $S_{\infty}^{1}$ is equal to that of $G^{\prime}$. Thus, $\bar{f}_{*}: G \longrightarrow G^{\prime}$ is the identity map as a transformation on $S_{\infty}^{1}$.

The $d$-neighborhood $\left\{y \in \mathbf{H}^{2}\right.$; $\left.\operatorname{dist}(y, J) \leq d\right\}$ of a closed subset $J$ of $\mathbf{H}^{2}$ is denoted by $\mathcal{N}_{d}\left(J, \mathbf{H}^{2}\right)$. When $J$ is a one point set $\{x\}, \mathcal{N}_{d}\left(\{x\}, \mathbf{H}^{2}\right)$ is also denoted by $B_{d}(x)$. For any geodesic line $\alpha$ in $\mathbf{H}^{2}, A_{\alpha}^{\natural}=\hat{\sigma}^{-1}(\alpha)$ is an open annulus properly 
embedded in $\hat{X}$. For $C>0$, we set $L_{C}(\alpha)=\hat{\sigma}^{-1}\left(\mathcal{N}_{C}\left(\alpha, \mathbf{H}^{2}\right)\right)$, which is a neighborhood of $A_{\alpha}^{\natural}$ in $\hat{X}$. An open annulus $A$ properly embedded in $\hat{X}$ is said to be a $\nu$-least area open annulus associated to $\alpha$ if $A$ satisfies the following two conditions:

- There exists $C>0$ with $A \subset L_{C}(\alpha)$ and such that $A$ is properly homotopic to $A_{\alpha}^{\natural}$ in $L_{C}(\alpha)$. The number $C$ is called a deviation of $A$.

- $A$ is $\nu$-least area. This means that any essential (compact) annulus $A_{0}$ in $A$ has the least area among all immersed annuli $A_{1}$ in $\hat{X}$ with $\partial A_{1}=\partial A_{0}$.

Lemma 2.1. For any geodesic line $\alpha \subset \mathbf{H}^{2}$, there exists a $\nu$-least area open annulus $A_{\alpha}$ associated to $\alpha$. Moreover, a deviation $C>0$ of $A_{\alpha}$ can be taken as a constant independent of $\alpha$.

Proof. The base orbifold $O(p, q, r)$ is divided by three geodesic segments $u_{1}, u_{2}, u_{3}$ into two hyperbolic triangles of interior angles $\pi / p, \pi / q, \pi / r$. The preimage $a^{-1}\left(u_{1} \cup u_{2} \cup u_{3}\right)$ is a union of finitely many closed geodesics $l_{1}, \cdots, l_{n}$ in $F$. Since $\pi_{1}(F)$ is residually finite, then replacing $F$ by a suitable finite covering of $F$, if necessary, we may assume that $l_{1}, \cdots, l_{n}$ are simple geodesics. Note that $\hat{a}^{-1}\left(l_{i}\right)$ is a disjoint union of geodesic lines in $\mathbf{H}^{2}$. Figure 2.1 below illustrates the union $\bigcup_{i=1}^{n} \hat{a}^{-1}\left(l_{i}\right)$. Let $\mathcal{L}$ be the set of all geodesic lines contained in $\bigcup_{i=1}^{n} \hat{a}^{-1}\left(l_{i}\right)$. The preimage $T_{i}^{\natural}=\sigma_{X}^{-1}\left(l_{i}\right)$ is an incompressible torus in $X$. According to FreedmanHass-Scott [4, there exists an embedded torus $T_{i}$ in $X$ which is $\nu$-least area among all tori homotopic to $T_{i}^{\natural}$. Each component $A_{i}$ of $\hat{p}^{-1}\left(T_{i}\right)$ is a $\nu$-least area open annulus associated to a component of $\hat{a}^{-1}\left(l_{i}\right)$.

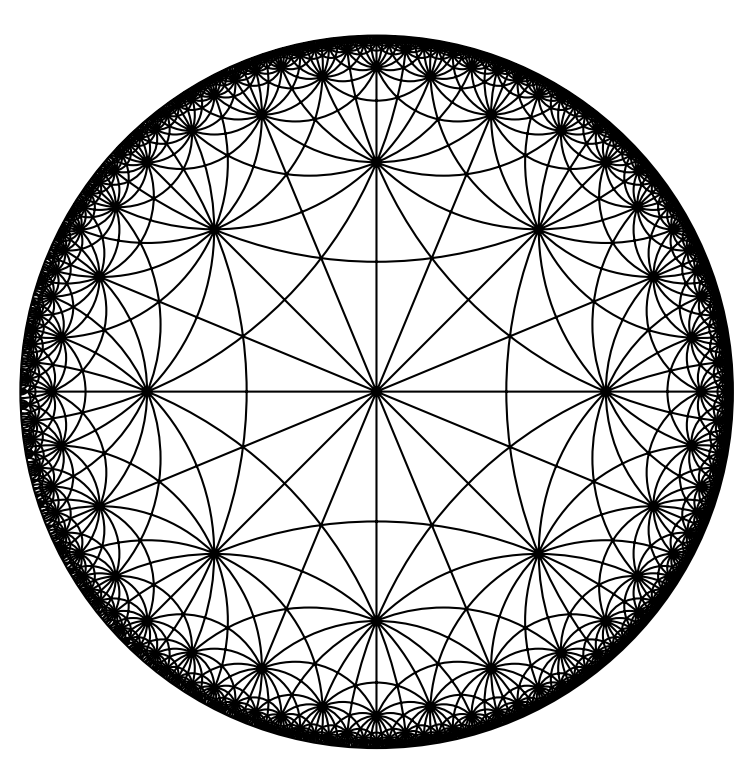

Figure 2.1. The case of $(p, q, r)=(2,3,8)$.

Since the $\nu$-length of each trace of the homotopy between $T_{i}$ and $T_{i}^{\natural}$ is uniformly bounded, that between $g^{\prime} A_{i}$ and $g^{\prime} A_{i}^{\natural}$ is uniformly bounded for any $g^{\prime} \in G^{\prime}$. Moreover, as is seen above, that between $g^{\prime} A_{i}^{\natural}$ and $g A_{i}^{\natural}$ is uniformly bounded. It 
follows that $g^{\prime} A_{i}$ 's $\left(g^{\prime} \in G^{\prime}, i=1, \cdots, n\right)$ have a common deviation $C_{1}>0$. We set $\mathcal{A}=\left\{g^{\prime} A_{i} ; g^{\prime} \in G^{\prime}, i=1, \cdots, n\right\}$. Consider a $G$-fundamental domain $D \subset \mathbf{H}^{2}$ of $O$ consisting of two hyperbolic triangles. Then, there exist finitely many geodesic lines $\lambda_{1}, \cdots, \lambda_{k} \in \mathcal{L}$ in $\mathbf{H}^{2}-D$ satisfying the following two conditions:

- For any $i \in\{1, \cdots, k\}, \lambda_{i}$ is disjoint from $\lambda_{j}$ 's $(j \neq i-1, i, i+1)$ and meets both $\lambda_{i-1}, \lambda_{i+1}$ transversely in single points, where $\lambda_{0}=\lambda_{k}$ and $\lambda_{k+1}=\lambda_{1}$.

- Let $\zeta, \eta$ be any geodesic lines in $\mathbf{H}^{2}$ meeting orthogonally in a single point of $D$. If $\eta$ meets $\lambda_{i}$ and $\lambda_{j}$ respectively in the distinct components of $\mathbf{H}^{2}-\zeta$, then $\mathcal{N}_{C_{1}}\left(\lambda_{u}, \mathbf{H}^{2}\right)$ is disjoint from $\zeta$ for any $u \in\{i-1, i, i+1, j-1, j, j+1\}$; see Figure 2.2(a).

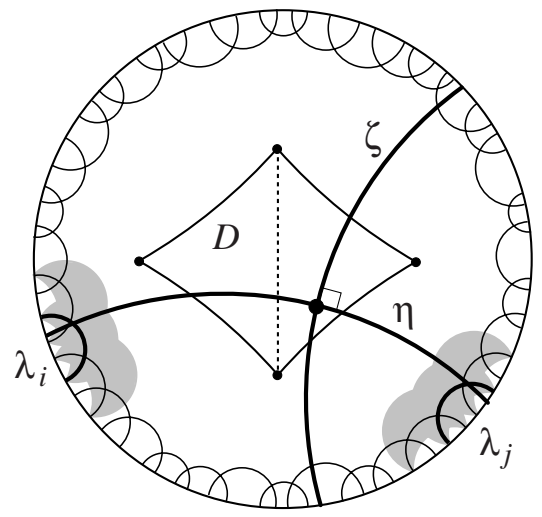

(a)

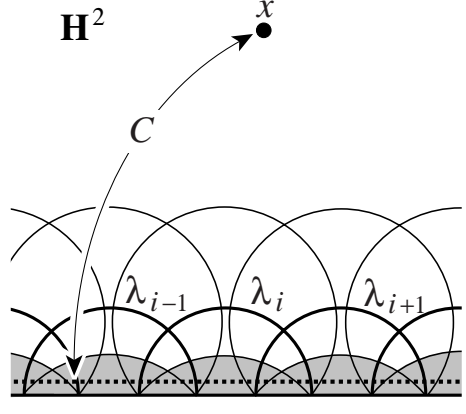

(b)

FIGURE 2.2. In (a), the two shaded regions represent respectively $\bigcup_{u=i-1}^{i+1} \mathcal{N}_{C_{1}}\left(\lambda_{u}, \mathbf{H}^{2}\right)$ and $\bigcup_{u=j-1}^{j+1} \mathcal{N}_{C_{1}}\left(\lambda_{u}, \mathbf{H}^{2}\right)$. In (b), the shaded region represents $Q_{1} \cup \cdots \cup Q_{k}$, and the dotted line represents $\partial B_{C}(x)$.

For $i \in\{1, \cdots, k\}$, the component of $\mathbf{H}^{2}-\operatorname{Int} \mathcal{N}_{C_{1}}\left(\lambda_{i}, \mathbf{H}^{2}\right)$ disjoint from $D$ is denoted by $Q_{i}$. There exists a constant $C>0$ with $\partial B_{C}(x) \subset \operatorname{Int}\left(Q_{1} \cup \cdots \cup Q_{k}\right)$ for any $x \in D$; see Figure 2.2 (b).

For any geodesic line $\alpha$ in $\mathbf{H}^{2}$, let $\mathcal{A}^{\vee}(\alpha)$ be the subset of $\mathcal{A}$ consisting of $A_{i} \in \mathcal{A}$ disjoint from the open annulus $A_{\alpha}^{\natural}=\hat{\sigma}^{-1}(\alpha)$. For $A_{i} \in \mathcal{A}^{\vee}(\alpha)$, the closure of the component of $\hat{X}-A_{i}$ disjoint from $A_{\alpha}^{\natural}$ is denoted by $E\left(A_{i}\right)$. Then, the union $E(\alpha)=\bigcup\left\{E\left(A_{i}\right) ; A_{i} \in \mathcal{A}^{\vee}(\alpha)\right\}$ consists of two components. For any geodesic line $\beta$ meeting $\alpha$ orthogonally in a single point $x \in \mathbf{H}^{2}$, consider an element $g \in G$ with $g(x) \in D$. The points $y, z \in g(\beta)$ of distance $C$ from $g(x)$ are contained respectively in $Q_{i-1} \cup Q_{i} \cup Q_{i+1}$ and $Q_{j-1} \cup Q_{j} \cup Q_{j+1}$, where $i, j$ are the numbers satisfying the above condition if we set $g(\alpha)=\zeta$ and $g(\beta)=\eta$. Then, $\hat{\sigma}^{-1}(\{y, z\})$ is contained in Int $E(g(\alpha))$. This implies that $L_{C}(\alpha)$ contains $\partial E(\alpha)$.

For convenience, we fix a direction of $\alpha$. Consider a sequence $\left\{\alpha_{0}, \alpha_{1}, \alpha_{2}, \cdots\right\}$ of compact connected subsets (geodesic segments) of $\alpha$ satisfying $\alpha_{n} \subset \operatorname{Int} \alpha_{n+1}$ and $\bigcup_{n} \alpha_{n}=\alpha$. The annulus $\Lambda_{n}=\hat{\sigma}^{-1}\left(\alpha_{n}\right)$ is a deformation retract of $A_{\alpha}^{\natural}$. 
For any $n \in \mathbf{N}$, let $\Lambda_{n}^{+}, \Lambda_{n}^{-}$be the components of $\Lambda_{n}-\operatorname{Int} \Lambda_{n-1}$, where $\Lambda_{n}^{+}$is supposed to be ahead of $\Lambda_{n}^{-}$with respect to the direction of $\alpha$. If necessary, replacing $\left\{\alpha_{n}\right\}$ by a suitable subsequence, one can have mutually disjoint elements $J_{n}^{\epsilon} \in \mathcal{A}$ $(\epsilon= \pm, n \in \mathbf{N})$ satisfying the following two conditions:

- $J_{n}^{\epsilon}$ meets $\Lambda_{n}^{\epsilon}$ non-trivially and $J_{n}^{\epsilon} \cap\left(A_{\alpha}^{\natural}-\operatorname{Int} \Lambda_{n}^{\epsilon}\right)$ is empty.

- The two end components of $J_{n}^{\epsilon}$ are contained in distinct components of $E(\alpha)$.

Let $\hat{X}_{n}$ be the closure of the middle component of $\hat{X}-J_{n}^{+} \cup J_{n}^{-}$, and set $Y_{n}(\alpha)=$ $\hat{X}_{n}-E(\alpha), \partial_{0} Y_{n}(\alpha)=\partial Y_{n}(\alpha) \cap\left(J_{n}^{+} \cup J_{n}^{-}\right)$; see Figure 2.3. By the Annulus Theorem (for example, see Jaco [9, Theorem VIII.13]), there exists an essential annulus $\left(A_{n ; \alpha}^{\natural}, \partial A_{n ; \alpha}^{\natural}\right)$ properly embedded in $\left(Y_{n}(\alpha), \partial_{0} Y_{n}(\alpha)\right)$. Let $A_{n ; \alpha}$ be a $\nu$-least area annulus in $\hat{X}_{n}$ bounding $\partial A_{n ; \alpha}^{\natural}$. Again by [4], $A_{n ; \alpha}$ is an embedded annulus. Since all elements of $\mathcal{A}^{\vee}(\alpha)$ are $\nu$-least area, $A_{n ; \alpha}$ is contained in $Y_{n}(\alpha)$. For any integer $m \geq n$, set $A_{n ; \alpha}^{(m)}=A_{m ; \alpha} \cap Y_{n}(\alpha)$. Then, $\left\{\left(A_{n ; \alpha}^{(m)}, \partial A_{n ; \alpha}^{(m)}\right)\right\}_{m \geq n}$ is a sequence of $\nu$-least area essential annuli properly embedded in $\left(Y_{n}(\alpha), \partial_{0} Y_{n}(\alpha)\right)$ with $\sup _{m}\left\{\operatorname{Area}_{\nu}\left(A_{n ; \alpha}^{(m)}\right)\right\} \leq \operatorname{Area}_{\nu}\left(\partial Y_{n}(\alpha)\right) / 2$. By the Ascoli-Arzelà Theorem, the sequence has a subsequence which converges uniformly to a $\nu$-least area annulus in $\left(Y_{n}(\alpha), \partial_{0} Y_{n}(\alpha)\right)$. By applying the diagonal argument to $\left\{A_{n, \alpha}\right\}$, one can show that the sequence has a subsequence converging uniformly on any compact set to a $\nu$-least area open annulus $A_{\alpha}$ properly embedded in $\bigcup_{n=1}^{\infty} Y_{n}(\alpha) \subset L_{C}(\alpha)$.

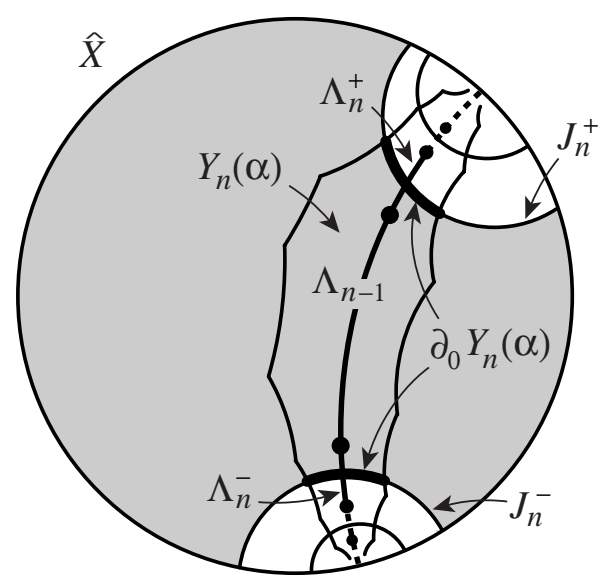

FiguRE 2.3. The shaded region represents $\hat{X}_{n}$.

Consider the $G$-orbit $G x=\left\{x_{i}\right\}$ of $x \in \mathbf{H}^{2}$. For any ordered pair $\left(x_{i}, x_{j}\right)$ of distinct points $x_{i}, x_{j} \in G x$, let $\alpha_{i: j}=\alpha_{j: i}$ be the perpendicular bisector line of the geodesic segment connecting $x_{i}$ with $x_{j}$, and let $H_{i: j}$ be the component of $\overline{\mathbf{H}^{2}}-\bar{\alpha}_{i: j}$ containing $x_{i}$. The closure $\delta_{i: j}$ of $S_{\infty}^{1} \cap H_{i: j}$ in $S_{\infty}^{1}$ is an arc satisfying $\delta_{i: j} \cup \delta_{j: i}=S_{\infty}^{1}$ and $\delta_{i: j} \cap \delta_{j: i}=\partial \delta_{i: j}=\partial \delta_{j: i}$.

The three $\operatorname{arcs} \delta_{1}, \delta_{2}, \delta_{3}$ in $S_{\infty}^{1}$ satisfy the tri-linking property if $\delta_{1} \cup \delta_{2} \cup \delta_{3}=S_{\infty}^{1}$. 
Definition 2.2. Under the notation above, the orbit $G x$ satisfies the insulator condition if, for each $x_{i} \in G x$, all triads $\delta_{j_{1}: i}, \delta_{j_{2}: i}, \delta_{j_{3}: i}$ do not have the tri-linking property.

Let $\Sigma_{O}$ be the singular point set of the hyperbolic triangle 2-orbifold $O=$ $O(p, q, r)$ with $2 \leq p \leq q \leq r$. The point of $\Sigma_{O}$ corresponding to an elliptic element of $G=\pi_{1}^{\text {orb }}(O)$ with order $r$ is denoted by $\bar{x}_{0}$.

The following lemma will play a crucial role in the proof of Lemma 3.1

Lemma 2.3. For any point $x_{0} \in(a \circ \hat{a})^{-1}\left(\left\{\bar{x}_{0}\right\}\right)$, the orbit $G x_{0}$ satisfies the insulator condition.

Proof. Let $\alpha_{0: j}$ be the perpendicular bisector line of the geodesic segment connecting $x_{0}$ with $x_{j} \in G x_{0}-\left\{x_{0}\right\}$, and let $d_{j}$ be the distance between $x_{0}$ and $\alpha_{0: j}$, that is, $d_{j}=\operatorname{dist}\left(x_{0}, x_{j}\right) / 2$. If $\delta_{j_{1}: 0}, \delta_{j_{2}: 0}, \delta_{j_{3}: 0}$ satisfied the tri-linking property, then $\Delta_{j_{1}, j_{2}, j_{3}}=\overline{H_{0: j_{1}} \cap H_{0: j_{2}} \cap H_{0: j_{3}}}$ would be the hyperbolic triangle containing $x_{0}$. The distances between $x_{0}$ and the three edges of $\Delta_{j_{1}, j_{2}, j_{3}}$ are $d_{j_{1}}, d_{j_{2}}, d_{j_{3}}$. In particular, $\Delta_{j_{1}, j_{2}, j_{3}}$ contains the round disk $D_{j_{1}, j_{2}, j_{3}}$ centered at $x_{0}$ of radius $d\left(x_{0}\right)=\min \left\{d_{j_{1}}, d_{j_{2}}, d_{j_{3}}\right\}$. Let $\Delta^{\prime}$ be a maximal hyperbolic triangle containing $\Delta_{j_{1}, j_{2}, j_{3}}$. Here, $\Delta^{\prime}$ being maximal means that all vertices of $\Delta^{\prime}$ are in $S_{\infty}^{1}$. Thus, $d\left(x_{0}\right)$ would not be greater than the inscribed radius of $\Delta^{\prime}$, which is $\log \sqrt{3}$ (for example see Beardon [1, §7.14]).

We will show that if $(p, q, r) \neq(2,3,7)$, then the inequality

$$
d\left(x_{0}\right)>\log \sqrt{3}=0.5493061 \cdots
$$

holds. As is suggested in Figure 2.4 if the inequality (2.1) holds for $(p, q, r)$, then it also holds for any $\left(p^{\prime}, q^{\prime}, r^{\prime}\right)$ with $p^{\prime} \geq p, q^{\prime} \geq q, r^{\prime} \geq r$. Thus, it suffices to consider the cases of $(p, q, r)=(2,3,8),(2,4,5),(3,3,4)$. From the formula on hyperbolic right-angled triangles given in [1, Theorem 7.11.3(i)], if $(p, q, r)=(2,3,8)$ or $(3,3,4)$, then

$$
d\left(x_{0}\right)=\operatorname{arccosh}\left(\frac{\cos (\pi / 3)}{\sin (\pi / 8)}\right)=0.7642854 \cdots
$$

holds; see Figure 2.5. Similarly, if $(p, q, r)=(2,4,5)$, then

$$
d\left(x_{0}\right)=\operatorname{arccosh}\left(\frac{\cos (\pi / 4)}{\sin (\pi / 5)}\right)=0.6268696 \cdots
$$

holds. Since in either case the inequality (2.1) holds, $G x_{0}$ satisfies the insulator condition whenever $(p, q, r) \neq(2,3,7)$.

On the other hand, if $(p, q, r)=(2,3,7)$, then

$$
d\left(x_{0}\right)=\operatorname{arccosh}\left(\frac{\cos (\pi / 3)}{\sin (\pi / 7)}\right)=0.5452748 \cdots
$$

is slightly smaller than $\log \sqrt{3}=0.5493061 \cdots$. If $d_{j_{1}}=d_{j_{2}}=d_{j_{3}}=d\left(x_{0}\right)$, then $\alpha_{0: j_{1}}, \alpha_{0: j_{2}}, \alpha_{0: j_{3}}$ contain distinct edges of the regular heptagon $H$ centered at $x_{0}$ as illustrated in Figure 2.6. It follows that $\delta_{j_{1}: 0}, \delta_{j_{2}: 0}, \delta_{j_{3}: 0}$ do not satisfy the trilinking property. Thus, at least one of $d_{j_{k}}(k=1,2,3)$, say $d_{j_{1}}$, is greater than 


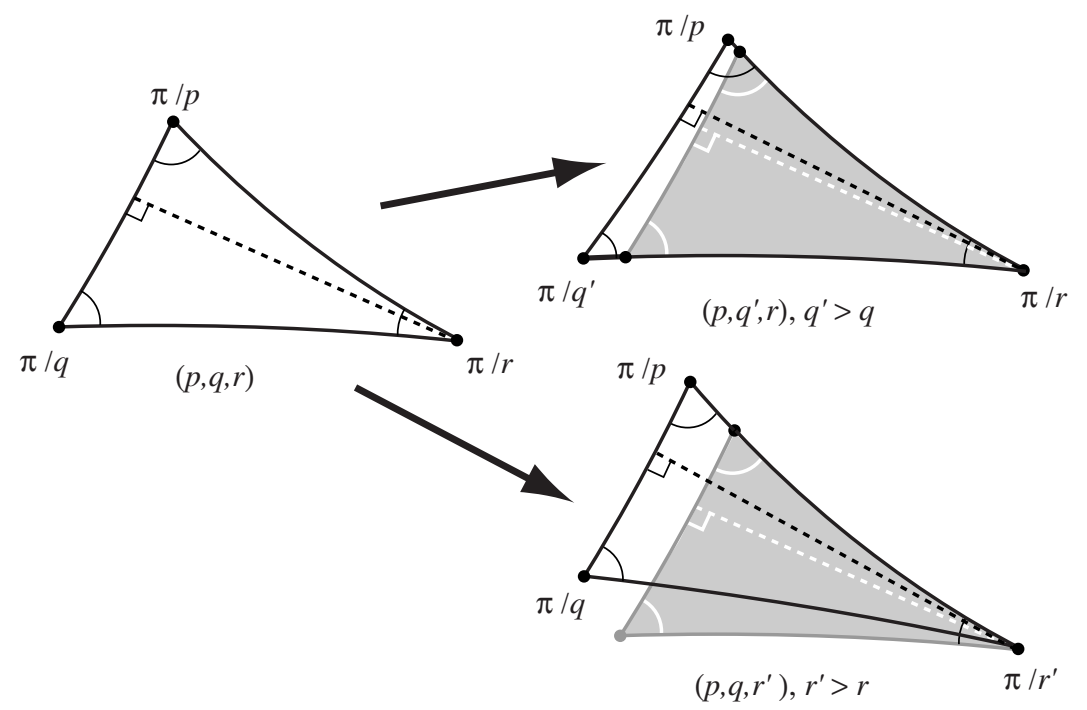

Figure 2.4. The cases of $q^{\prime}>q$ and $r^{\prime}>r$. The situation in the case of $p^{\prime}>p$ is similar to that of $q^{\prime}>q$.
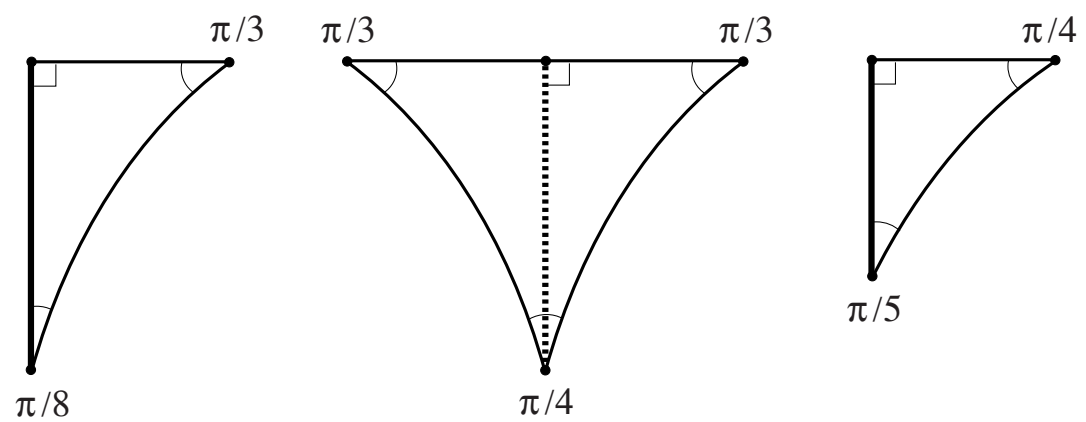

FIGURE 2.5.

$d\left(x_{0}\right)$. Then, we have

$$
d_{j_{1}} \geq \frac{1}{2} \operatorname{dist}\left(x_{0}, x_{8}\right)=\operatorname{arccosh}\left(\frac{\cos (2 \pi / 7)}{\sin (\pi / 7)}\right)=0.9037998 \cdots,
$$

where $x_{8}$ is the point in $\mathbf{H}^{2}$ as shown in Figure 2.6. Suppose that $\delta_{j_{1}: 0}, \delta_{j_{2}: 0}, \delta_{j_{3}: 0}$ satisfied the tri-linking property. Since the radius $d\left(x_{0}\right)$ of $D_{j_{1}, j_{2}, j_{3}} \subset \Delta_{j_{1}, j_{2}, j_{3}}$ has a value sufficiently near to $\log \sqrt{3}, \Delta_{j_{1}, j_{2}, j_{3}}$ would be geometrically well approximated by $\Delta^{\prime}$. From this, we know that $\Delta_{j_{1}, j_{2}, j_{3}}$ does not have any edge with $d_{j_{k}}>0.9$, a contradiction. In fact, it is not hard to show that $d_{j_{k}}$ is at most $0.55739 \ldots$ by considering the extremal case when a round disk of radius $d\left(x_{0}\right)$ touches two edges of $\Delta^{\prime}$. Thus, also in the case of $(p, q, r)=(2,3,7), G x_{0}$ satisfies the insulator condition. 


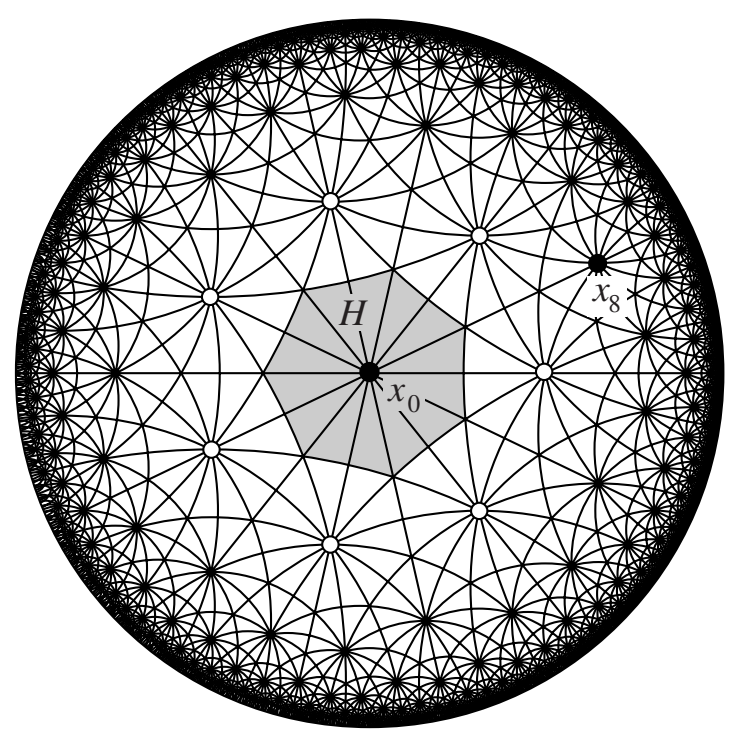

Figure 2.6. The seven white dots represent the points of $G x_{0}$ nearest to $x_{0}$, and $x_{8}$ represents one of the points of $G x_{0}$ next to these seven points.

\section{Proofs of TheOREMS}

We will always assume that any two least area surfaces meet each other transversely, if necessary by deforming them slightly. Fix an $x_{0} \in(a \circ \hat{a})^{-1}\left(\bar{x}_{0}\right)$ for the point $\bar{x}_{0} \in \Sigma_{O}$ given in Lemma 2.3. Set $G x_{0}=\left\{x_{i}\right\}_{i \in \Gamma}$ for a countable set $\Gamma$. As in 22, for any ordered pair $(i, j)$ of distinct elements of $\Gamma$, let $\alpha_{i: j}=\alpha_{j: i}$ be the perpendicular bisector line of the geodesic segment connecting $x_{i}$ with $x_{j}$. Let $A_{i: j}$ be a $\nu$-least area open annulus associated to $\alpha_{i: j}$. We will take them so that $g^{\prime} A_{i: j}=A_{u: v}$ if $g\left(x_{i}\right)=x_{u}, g\left(x_{j}\right)=x_{v}$ for $g \in G$. Note that then $A_{i: j} \neq A_{j: i}$ may occur. If $\alpha_{i: j}$ and $\alpha_{k: l}$ do not have common end points, then by the $\nu$-least area property for $A_{i: j}$ and $A_{k: l}$, the intersection $A_{i: j} \cap A_{k: l}$ is either empty or a single essential loop. If $\alpha_{i: j}$ and $\alpha_{k: l}$ has only one common end point, then $A_{i: j} \cap A_{k: l}$ has at most one compact component (an essential loop) and any other component $\xi$ is a properly embedded arc in $A_{i: j}$ (resp. $A_{k: l}$ ) such that one of the two components of $A_{i: j}-\xi$ (resp. $A_{k: l}-\xi$ ) is an open disk. Note that the two end components of $\xi$ are contained in a single end component of $A_{i: j}$. Similarly, if $\alpha_{i: j}=\alpha_{k: l}$, then either $A_{i: j}=A_{k: l}$ or $A_{i: j} \cap A_{k: l}$ consists of at most one essential loop and properly embedded arcs $\xi$ each of which either excises from $A_{i: j}$ and $A_{k: l}$ open disks or cuts them into single open disks. The latter case occurs when $\xi$ is a curve connecting the distinct end components of these annuli. The component of $\hat{X}-A_{i: j}$ adjacent to $\delta_{i: j} \subset \partial \hat{X}=S_{\infty}^{1}\left(\right.$ resp. $\left.\delta_{j: i}\right)$ is denoted by $\hat{H}_{i: j}^{+}\left(\right.$resp. $\left.\hat{H}_{i: j}^{-}\right)$. Note that in the case when $A_{i: j} \neq A_{j: i}, \hat{H}_{i: j}^{+}$is not equal to $\hat{H}_{j: i}^{-}$.

A simple loop $c$ in an open solid torus $V$ is called a core if $V-c$ is homeomorphic to $T^{2} \times(0,1)$. 
Lemma 3.1. For any $i \in \Gamma$, the intersection $\bigcap_{j \in \Gamma-\{i\}}\left(\hat{H}_{i: j}^{+} \cap \hat{H}_{j: i}^{-}\right)$has only one component $\hat{V}_{i}$ which is an open solid torus such that a core of $\hat{V}_{i}$ is also a core of $\hat{X}$. Moreover, any other component of $\bigcap_{j \in \Gamma-\{i\}}\left(\hat{H}_{i: j}^{+} \cap \hat{H}_{j: i}^{-}\right)$is an open 3-ball.

Proof. By Lemma 2.1, one can have a common deviation for all $A_{i: j}$ 's. Thus, there exist finitely many $\hat{H}_{k}$ 's $(k=1, \cdots, m)$ with either $\hat{H}_{k}=\hat{H}_{i: j_{k}}^{+}$or $\hat{H}_{k}=\hat{H}_{j_{k}: i}^{-}$and such that

$$
\bigcap_{j \in \Gamma-\{i\}}\left(\hat{H}_{i: j}^{+} \cap \hat{H}_{j: i}^{-}\right)=\hat{H}_{1} \cap \cdots \cap \hat{H}_{m} .
$$

We set $A_{k}=\partial \hat{H}_{k}$. Then, $A_{k}$ is either $A_{i: j_{k}}$ or $A_{j_{k}: i}$. Suppose that $\mathcal{H}_{u-1}=$ $\hat{H}_{1} \cap \cdots \cap \hat{H}_{u-1}$ satisfies the assertion of this lemma; we will show that $\mathcal{H}_{u}$ also satisfies it. Since $A_{u}$ is $\nu$-least area, each component $J$ of $A_{u} \cap \mathcal{H}_{u-1}$ is ether an open disk or an open annulus. Moreover, in the latter case, a simple essential loop in $J$ is also essential in $A_{u}$. As is shown in Figure 3.1, $A_{u} \cap \mathcal{H}_{u-1}$ has at most one open annulus component $J$. The figure illustrates the case when $\partial_{-} J$ and $\partial_{+} J$ meet respectively a compact component $\lambda_{-}$of $A_{u} \cap A_{k}$ and a non-compact component $\lambda_{+}$of $A_{u} \cap A_{l}$ for some $k, l$ with $1 \leq k, l \leq u-1$. The $\lambda_{-}$is an essential loop and $\lambda_{+}$is a properly embedded curve in $A_{u}$. Note that the left side component of $A_{u}-\lambda_{-}$is contained in $\operatorname{Int}\left(\hat{X}-\hat{H}_{k}\right) \subset \operatorname{Int}\left(\hat{X}-\mathcal{H}_{u-1}\right)$.

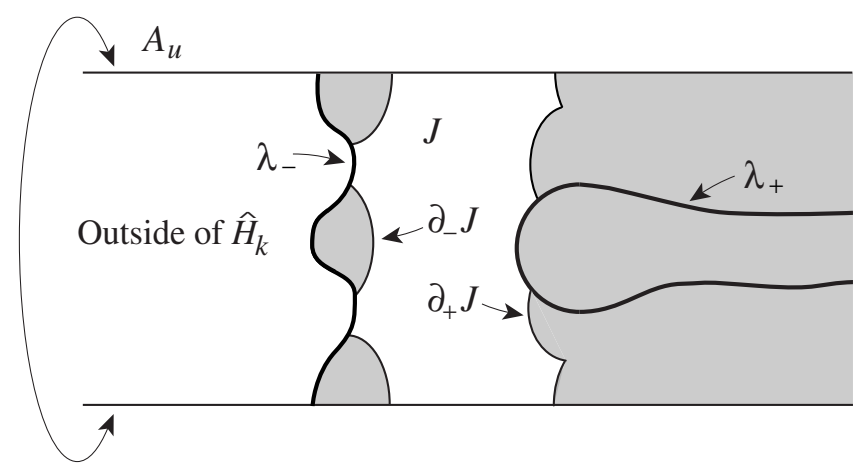

Figure 3.1. $A_{u} \cap \mathcal{H}_{u-1}-J$ is contained in the shaded part, which is a disjoint union of open disks. Thus, any component of $A_{u} \cap \mathcal{H}_{u-1}$ other than $J$ is an open disk.

A core $c$ of the open solid torus component $V$ of $\mathcal{H}_{u-1}$ meets non-trivially only finitely many open disk components $\Delta_{v}$ of $A_{u} \cap \mathcal{H}_{u-1}$. If the intersection number $\left[\Delta_{v}\right] \cdot[c] \neq 0$, that is, $\Delta_{v}$ were a 'meridian disk' of $V$, then $V-\Delta_{v}$ would be an open ball. It follows that all components of $\mathcal{H}_{u}$ would be open 3-balls. As in [6, Lemma 4.3], one can show that some triad $\delta_{w_{1}: i}, \delta_{w_{2}: i}, \delta_{w_{3}: i}$ would have the tri-linking property. This contradicts the fact that $G x_{0}$ satisfies the insulator condition by Lemma 2.3. Thus, each open disk $\Delta_{v}$ excises from $V$ an open 3-ball $B_{v}$. Deforming $c$ by an ambient isotopy with its support a small regular neighborhood of $\bigcup_{v} B_{v}$, one can cancel the intersection $c \cap\left(\bigcup_{v} \Delta_{v}\right)$. This shows that the union of open disk components of $A_{u} \cap \mathcal{H}_{u-1}$ divides $V$ into one open solid torus $V^{\prime}$ and open 3-balls. Moreover, a core $c^{\prime}$ of $V^{\prime}$ is isotopic to $c$ in $V$ and hence $c^{\prime}$ is a core of $\hat{X}$. In the case when $A_{u} \cap \mathcal{H}_{u-1}$ contains an open annulus component $J, J$ divides $V^{\prime}$ into 
two open solid tori $V_{1}^{\prime}$ and $V_{2}^{\prime}$. One of them, say $V_{1}^{\prime}$, is contained in $\hat{H}_{u}$ and hence in $\mathcal{H}_{u}$, but the other is disjoint from $\hat{H}_{u}$. Thus, $V_{1}^{\prime}$ is a unique open solid torus component of $\mathcal{H}_{u}$. A simple essential loop $c_{J}$ of $J$ is a core of $A_{u}$ and hence that of $\hat{X}$. A simple loop $c_{1}^{\prime}$ of $V_{1}^{\prime}$ isotopic to $c_{J}$ in $\overline{V_{1}^{\prime}}$ is a core of both $V_{1}^{\prime}$ and $\hat{X}$.

Repeating the same argument, one can show that $\mathcal{H}_{m}=\bigcap_{j \in \Gamma-\{i\}}\left(\hat{H}_{i: j}^{+} \cap \hat{H}_{j: i}^{-}\right)$ satisfies the required conditions.

Proof of Theorem 0.1. From the definition of open solid tori $\hat{V}_{i}(i \in \Gamma)$ given in Lemma 3.1, they are mutually disjoint and $G^{\prime}$-equivariant. In particular, for $g^{\prime} \in G^{\prime}$ corresponding to any element $g$ of the stabilizer $\operatorname{Stab}_{G}\left(\bar{x}_{0}\right) \cong \mathbf{Z}_{r}$, we have $g^{\prime} \hat{V}_{0}=\hat{V}_{0}$. Then, there exists a $G^{\prime}$-equivariant solid torus $\hat{W}_{0} \subset \hat{V}_{0}$ such that a core of $\hat{W}_{0}$ is a core of $\hat{X}$. The image $W_{0}=q_{1} \circ \hat{p}\left(\hat{W}_{0}\right)$ is a solid torus in $N$. Set $\hat{W}_{i}=g^{\prime} \hat{W}_{0}$ for $g \in G$ with $g\left(x_{0}\right)=x_{i}$. Since a core of any $\hat{W}_{i}$ is a core of $\hat{X}$ and since any two $\hat{W}_{i}$ and $\hat{W}_{j}$ are separated by the open annulus $A_{i: j}, \bigcup_{i} \hat{W}_{i}$ is an unknotted 'closed braid' in the open solid torus $\hat{X}$, that is, $\hat{Y}=\hat{X}-\bigcup_{i} \operatorname{Int} \hat{W}_{i}$ is homeomorphic to $R \times S^{1}$ for some planar surface $R$ such that $\pi_{1}(R)$ is an infinitely generated free group. From $\pi_{1}(\hat{Y}) \cong \pi_{1}(R) \times \mathbf{Z}$, we know that $\pi_{1}(\hat{Y})$ has the center $Z=\langle h\rangle$ isomorphic to $\mathbf{Z}$. Since $q_{1} \circ \hat{p} \mid \hat{Y}: \hat{Y} \longrightarrow N-\operatorname{Int} W_{0}$ is the restriction of the regular covering $q_{1} \circ \hat{p}$, it is also a regular covering. Thus, $\pi_{1}(\hat{Y})$ can be regarded as a normal subgroup of $\pi_{1}\left(N-\operatorname{Int} W_{0}\right)$. In particular, for any $g \in \pi_{1}\left(N-\operatorname{Int} W_{0}\right)$, $g h g^{-1}$ is an element of $\pi_{1}(\hat{Y})$. Moreover, for any $f \in \pi_{1}(\hat{Y})$, we have

$$
\begin{aligned}
f\left(g h g^{-1}\right) f^{-1} & =g\left[\left(g^{-1} f g\right) h\left(g^{-1} f g\right)^{-1}\right] g^{-1} \\
& =g\left[h\left(g^{-1} f g\right)\left(g^{-1} f g\right)^{-1}\right] g^{-1}=g h g^{-1} .
\end{aligned}
$$

This implies that $g h g^{-1} \in Z$, and hence $Z$ is a normal subgroup of $\pi_{1}\left(N-\operatorname{Int} W_{0}\right)$. Since $N-\operatorname{Int} W_{0}$ is a Haken manifold, this shows that $N-\operatorname{Int} W_{0}$ admits a Seifert fibration (see [9, Theorem VI.24]). Thus, $N$ has a Seifert fibration extending that on $N-\operatorname{Int} W_{0}$. Since then $f: M \longrightarrow N$ is a homotopy equivalence between closed Seifert fibered spaces of infinite $\pi_{1}, f$ is homotopic to a homeomorphism.

Proof of Theorem 0.2. Let $f: M \longrightarrow M$ be a self-homeomorphism of a closed Seifert fibered space $M$ with the hyperbolic base 2-orbifold $O$ which is homotopic to the identity $\operatorname{Id}_{M}$ of $M$. If $O$ is not a triangle orbifold, then $M$ is a Haken manifold, and hence the proof of Theorem 0.2 is reduced to Waldhausen [14, Theorem 7.1]. So, we may assume that $O$ is a triangle 2-orbifold $O(p, q, r)$ with $2 \leq p \leq q \leq r$, $1 / p+1 / q+1 / r<1$. Moreover, one can suppose that $f$ is a diffeomorphism. Fix a Riemannian metric $\nu$ on $M$. Then, $f_{*}(\nu)$ is another Riemannian metric on $M$. Consider a solid torus $W_{\nu}$ in $M$ defined as $W_{0} \subset N$ in the proof of Theorem 0.1 with respect to $\nu$. Note that $W_{\nu}$ contains an exceptional fiber in $M$ of order $r$. The other two exceptional fibers are contained in $M-\operatorname{Int} W_{\nu}$. Similarly, $f\left(W_{\nu}\right)$ can be regarded as a solid torus $W_{f_{*}(\nu)}$ in $M$ with respect to $f_{*}(\nu)$.

First, we will show that $\operatorname{Id}_{M}$ is isotopic to a diffeomorphism $f^{\prime}: M \longrightarrow M$ with $f^{\prime}\left(W_{\nu}\right)=f\left(W_{\nu}\right)$. Let us consider a continuous deformation of Riemannian metrics on $M$ from $\nu=\nu_{0}$ to $f_{*}(\nu)=\nu_{1}$ defined as follows. Let $g_{0}, g_{1}$ be the inner products on the tangent bundle $T(M)$ determining the Riemannian metrics $\nu_{0}, \nu_{1}$. Then, the Riemannian metric $\nu_{t}(t \in[0,1])$ on $M$ is determined by the inner product $g_{t}$ 
on $T(M)$ such that

$$
g_{t}(u, v)=(1-t) g_{0}(u, v)+t g_{1}(u, v)
$$

for any $x \in M$ and any two vectors $u, v \in T_{x}(M)$. Let $\hat{V}_{i[t]}$ be a $G$-equivariant open solid torus in $\hat{X}$ defined as in Lemma 3.1 with respect to $\nu_{t}$, and let $W_{t}$ be an unknotted solid torus in the open solid torus $p_{1} \circ \hat{p}\left(\hat{V}_{i[t]}\right) \subset M$. For the proof, it suffices to see that any $t_{0} \in[0,1]$ has a neighborhood $U$ such that, for each $t \in U$, $W_{t}$ is ambient isotopic to $W_{t_{0}}$ in $M$. If not, there would exist a sequence $\left\{t_{n}\right\}$ in $[0,1]$ converging to $t_{0}$ such that any $W_{t_{n}}$ is not ambient isotopic to $W_{t_{0}}$. Let $l_{1}, \cdots, l_{m}$ be simple closed geodesics in $F$ as in the proof of Lemma 2.1. Let $T_{k\left[t_{n}\right]}$ be a $\nu_{t_{n}}$-least area torus homotopic to the embedded torus $T_{k}^{\natural}=\sigma_{X}^{-1}\left(l_{k}\right)$ in $X$. Note that the $\nu_{t_{0}}$-areas of $T_{k\left[t_{n}\right]}$ 's are bounded from above. In fact, from (3.1), we have a constant $K \geq 1$ independent of $n$ such that

$$
\operatorname{Area}_{t_{0}}\left(T_{k\left[t_{n}\right]}\right) \leq K \operatorname{Area}_{t_{n}}\left(T_{k\left[t_{n}\right]}\right) \leq K \operatorname{Area}_{t_{n}}\left(T_{k}^{\natural}\right) \leq K^{2} \operatorname{Area}_{t_{0}}\left(T_{k}^{\natural}\right) .
$$

By the Ascoli-Arzelà Theorem, if necessary passing to a subsequence of $\left\{t_{n}\right\}$, we may assume that each sequence $\left\{T_{k\left[t_{n}\right]}\right\}_{n=1}^{\infty}$ converges uniformly to a $\nu_{t_{0}}$-least area torus $T_{k\left[t_{0}\right]}^{\bullet}$. Note that either $T_{k\left[t_{0}\right]}=T_{k\left[t_{0}\right]}^{\bullet}$ or $T_{k\left[t_{0}\right]} \cup T_{k\left[t_{0}\right]}^{\bullet}$ bounds a submanifold of $X$ homeomorphic to $T^{2} \times I$. From this convergence, one can take a constant $C_{1}>0$ independent of $n$ as a deviation of any component $A_{k\left[t_{n}\right]}$ of $\hat{p}^{-1}\left(T_{k\left[t_{n}\right]}\right)$. Then, as in the proof of Lemma 2.1. we have a constant $C>0$ independent of $n$ as a deviation of a $\nu_{t_{n}}$-least area open annulus $A_{\alpha\left[t_{n}\right]}$ associated to any geodesic line $\alpha$ in $\mathbf{H}^{2}$. Again passing to a subsequence of $\left\{t_{n}\right\}$ if necessary, we may assume that the sequence $\left\{A_{i: j\left[t_{n}\right]}\right\}_{n=1}^{\infty}$ converges uniformly on all compact subsets to a $\nu_{t_{0}}$-least area open annulus $A_{i: j\left[t_{0}\right]}^{\bullet}$ associated to $\alpha_{i: j}$. As in Lemma 3.1. $A_{i: j\left[t_{0}\right]}^{\bullet}$ and $A_{j: i\left[t_{0}\right]}^{\bullet}$ determine a $G$-equivariant open solid torus $\hat{V}_{i\left[t_{0}\right]}^{\bullet}$. Let $W_{t_{0}}^{\bullet}$ be an unknotted solid torus in $p_{1} \circ \hat{p}\left(\hat{V}_{i\left[t_{0}\right]}^{\bullet}\right)$. From the uniform convergence of $\left\{A_{i: j\left[t_{n}\right]}\right\} \rightarrow A_{i: j\left[t_{0}\right]}^{\bullet}$ in compact subsets, we know that $W_{t_{n}}$ is ambient isotopic to $W_{t_{0}}^{\bullet}$ in $M$ for any $n \in \mathbf{N}$ greater than a sufficiently large $n_{0}$. Applying the argument in the proof of Lemma 3.1 to $A_{i: j\left[t_{0}\right]}, A_{j: i\left[t_{0}\right]}, A_{i: j\left[t_{0}\right]}^{\bullet}, A_{j: i\left[t_{0}\right]}^{\bullet}(j \in \Gamma-\{i\})$, one can show that $\hat{V}_{i\left[t_{0}\right]} \cap \hat{V}_{i\left[t_{0}\right]}^{\bullet}$ has a unique open solid torus component $\hat{V}_{i\left[t_{0}\right]}^{\mathbf{\Delta}}$, a core of which is also a core of $\hat{X}$. This implies that a core of $\hat{V}_{i\left[t_{0}\right]}$ (resp. of $\left.\hat{V}_{i\left[t_{0}\right]}^{\bullet}\right)$ is isotopic to a core of $\hat{V}_{i\left[t_{0}\right]}^{\mathbf{\Delta}}$ in $\hat{V}_{i\left[t_{0}\right]}$ (resp. in $\hat{V}_{i\left[t_{0}\right]}^{\bullet}$ ). It follows that $W_{t_{0}}^{\bullet}$ and hence $W_{t_{n}}\left(n>n_{0}\right)$ are ambient isotopic to $W_{t_{0}}$ in $M$. This contradiction shows the existence of our desired diffeomorphism $f^{\prime}$.

Since the Seifert fibration on $M-\operatorname{Int} W_{\nu}$ has the base orbifold with a disk as its underlying space and with two exceptional fibers, $M-\operatorname{Int} W_{\nu}$ has a unique essential annulus $A$ up to ambient isotopy. Thus, $f^{\prime}$ is isotopic to $f^{\prime \prime}$ with $f^{\prime \prime}\left(W_{\nu}\right)=f\left(W_{\nu}\right)$ and $f^{\prime \prime}(A)=f(A)$. Moreover, we may assume that, for some essential simple loop $c$ in $\operatorname{Int} A, f^{\prime \prime}|c=f| c$. If $f^{-1} \circ f^{\prime \prime} \mid A: A \longrightarrow A$ were an orientation-reversing homeomorphism, then the homomorphism $\bar{f}_{*}: G \longrightarrow G$ induced from the identity $\operatorname{Id}_{\pi_{1}(M)}=f_{*}: \pi_{1}(M) \longrightarrow \pi_{1}(M)$ would exchange elliptic elements corresponding to the two exceptional fibers in $M-\operatorname{Int} W_{\nu}$, a contradiction. This shows that $f^{\prime \prime}$ is isotopic to $f^{\prime \prime \prime}$ with $f^{\prime \prime \prime}|(T \cup A)=f|(T \cup A)$, where $T=\partial W_{\nu}$. Since the closure of each component of $M-T \cup A$ is a solid torus, $f^{\prime \prime \prime}$ is isotopic to $f$. This completes the proof. 


\section{ACKNOWLEDGEMENT}

The author thanks the referee for valuable comments and suggestions. He also thanks Sadayoshi Kojima and Shigeru Mizushima for offering him the originals of Figures 2.1 and 2.6 .

\section{REFERENCES}

[1] A. Beardon, The geometry of discrete groups, Graduate Texts in Math. 91, Springer-Verlag (1983). MR0698777 (85d:22026)

[2] M. Boileau and J.-P. Otal, Groupe des difféotopies de certaines variétés de Seifert, C. R. Acad. Sci. Paris Ser. I Math. 303 (1986) 19-22. MR0849619 (87g:57022)

[3] A. Casson and D. Jungreis, Convergence groups and Seifert fibered 3-manifolds, Invent. Math. 118 (1994) 441-456. MR1296353 (96f:57011)

[4] M. Freedman, J. Hass and P. Scott, Least area incompressible surfaces in 3-manifolds, Invent. Math. 71 (1983), 609-642. MR0695910 (85e:57012)

[5] D. Gabai, Convergence groups are Fuchsian groups, Ann. of Math. 136 (1992) 447-510. MR.1189862 (93m:20065)

[6] D. Gabai, On the geometric and topological rigidity of hyperbolic 3-manifolds, J. Amer. Math. Soc. 10 (1997) 37-74. MR1354958 (97h:57028)

[7] D. Gabai, R. Meyerhoff and N. Thurston, Homotopy hyperbolic 3-manifolds are hyperbolic, Ann. of Math. 157 (2003) 335-431. MR.1973051 (2004d:57020)

[8] J. Hempel, 3-manifolds, Annals of Mathematics Studies 86, Princeton Univ. Press (1976). MR0415619(54:3702)

[9] W. Jaco, Lectures on three-manifold topology, C.B.M.S. Regional Conf. Ser. in Math. no. 43, Amer. Math. Soc. (1980). MR0565450 (81k:57009)

[10] W. Meeks III, L. Simon and S.-T. Yau, Embedded minimal surfaces, exotic spheres, and manifolds with positive Ricci curvature, Ann. of Math. 116 (1982) 621-659. MR0678484 (84f:53053)

[11] P. Scott, There are no fake Seifert fibre spaces with infinite $\pi_{1}$, Ann. of Math. 117 (1983) 35-70. MR0683801 (84c:57008)

[12] P. Scott, Homotopy implies isotopy for some Seifert fibre spaces, Topology 24 (1985) 341-351. MR0815484 (87c:57012)

[13] T. Soma, Existence of least area planes in hyperbolic 3-space with co-compact metric, Topology 43 (2004) 705-716. MR2041639(2005a:57017)

[14] F. Waldhausen, On irreducible 3-manifolds which are sufficiently large, Ann. of Math. 87 (1968) 56-88. MR0224099(36:7146)

Department of Mathematical Sciences, College of Science and Engineering, Tokyo Denki University, Hatoyama-machi, Saitama-Ken 350-0394, Japan

E-mail address: soma@r.dendai.ac.jp 